\title{
Amnioinfusão em Trabalho de Parto com Líquido Meconiado
}

\author{
Amnioinfusion during Labor with Meconium-stained Amniotic Fluid
}

Ricardo F. Savaris

\begin{abstract}
RESUMO
Objetivo: relatar a experiência com o uso da técnica de amnioinfusão nas pacientes em trabalho de parto que apresentam mecônio e verificar a incidência de complicações, da sindrome da aspiração do mecônio com o uso desse procedimento e de cesáreas.

Método: foram estudadas retrospectivamente 20 gestantes a termo em trabalho de parto, com líquido amniótico meconiado no Centro Obstétrico de duas instituições do Sistema Único de Saúde no Estado do Rio Grande do Sul. A infusão inicial de $1000 \mathrm{ml}$ de soro fisiológico não-aquecido foi feita a velocidade de 20 a $30 \mathrm{ml}$ por minuto, depois reduzida para $3 \mathrm{ml}$ por minuto. O líquido era drenado com a elevação da apresentação cefálica.

Resultados: a amnioinfusão demonstrou-se de fácil aplicação, quando foi utilizado um cateter nasogástrico. Nenhuma paciente apresentou complicações importantes relacionadas com o procedimento. Não houve presença de mecônio abaixo das cordas vocais nos recém-nascidos. A incidência de parto cesárea foi de 3/20 (15\%).

Conclusões: a amnioinfusão é uma técnica de baixo custo e factivel que não apresentou complicações nos casos relatados.
\end{abstract}

PALAVRAS-CHAVE: Amnioinfusão. Sofrimento fetal. Trabalho de parto.

\section{Introdução}

A descrição da instilação de soro fisiológico da cavidade uterina é relativamente recente, tendo sido publicada pela primeira vez em $1983^{1}$. Desde então, essa técnica, de fácil aplicação e de baixo custo, tem sido investigada sob diferentes aspectos.

A amnioinfusão tem se demonstrado benéfica para melhorar a presença de oligoidrâmnio ${ }^{2}$, desacelerações variáveis ou tardias ${ }^{3}$ e nos casos em que há mecônio na cavidade amniótica ${ }^{4}$.

A presença de mecônio durante o trabalho de parto é um sinal de alerta para o obstetra. As complicações oriundas dessa condição podem aumentar a incidência de parto cesáreo e a sindrome da aspiração de mecônio no recémnascido ${ }^{5}$. O uso de soro fisiológico durante o Hospital Bartholomeu Tacchini - Bento Gonçalves - Rio Grande do Sul

Hospital Municipal de Santo Antônio da Patrulha - Rio Grande do Sul

Correspondência:

Ricardo Savaris

Rua Reis Louzada,33

90630-130 - Porto Alegre - RS

e.mail: savaris@orion.ufrgs.br trabalho de parto na presença de mecônio já foi descrito na literatura ${ }^{3,4}$. Foi demonstrado, também, que não há necessidade de aquecer o soro para o uso no intraparto ${ }^{6}$. Esse método, apesar de não ser dispendioso, ainda não está difundido em nosso meio. A sua utilização no Brasil está restrita ao tratamento e diagnóstico de oligoidrâmnio e da rotura prematura das membranas ${ }^{7}$.

Cecatti et $a .^{7}$, numa recente revisão, sugeriram a utilização de uma sonda de Foley ou de um cateter de pressão intra-uterina, durante o trabalho de parto para fazer a infusão do soro aquecido. Esses mesmos autores, após a revisão da literatura disponivel, verificaram que a amnioinfusão parece ser um procedimento de valor para o tratamento da diluição do mecônio durante o trabalho de parto ${ }^{7}$. Logo, por se tratar de uma técnica de baixo custo, de fácil aplicabilidade e com potenciais beneficios para as gestantes em trabalho de parto, foi recomendada a incorporação de tal técnica no manejo obstétrico. Para isso, é necessário realizar estudos para verificar a aplicabilidade dessa técnica dentro do contexto brasileiro.

O objetivo do presente trabalho é relatar a 
experiência pessoal no uso dessa técnica e a incidência de complicações, de cesáreas e da sindrome da aspiração do mecônio.

\section{Pacientes e Métodos}

Foram avaliadas no presente trabalho dez pacientes admitidas no Centro Obstétrico do Hospital Bartholomeu Tacchini, no periodo de janeiro e novembro de 1997, e dez pacientes admitidas no Centro Obstétrico do Hospital Municipal de Santo Antônio da Patrulha, durante o período de fevereiro a junho de 1998 (ambos no Rio Grande do Sul), todas sob os cuidados do autor. Foram incluídas as pacientes com idade gestacional superior a 37 semanas, sem cesárea prévia, em trabalho de parto ativo, com dilatação cervical $>4 \mathrm{~cm}$, feto único, em apresentação cefálica, apresentando líquido meconiado (leve a espesso). Foi considerado mecônio leve, quando o líquido apresentava um aspecto esverdeado e aquoso; moderado, quando o líquido amniótico apresentava-se esverdeado, opaco, não-aquoso, e mecônio espesso, quando o mesmo tinha um aspecto de "sopa-de-ervilha".

Foram excluídas as pacientes com fetos com apresentação pélvica, as que apresentavam sinais de infecção ovular e sofrimento fetal agudo no momento da internação.

A amnioscopia ou o exame especular foi realizado no momento da admissão hospitalar. A ruptura artificial das membranas e a monitorização fetal foram realizadas após a identificação do líquido amniótico meconiado. Um cateter nasogástrico $\mathrm{n}^{\circ} 14$ foi conectado a um frasco de $1000 \mathrm{ml}$ de soro fisiológico por meio de um equipo de soro. Sob condições assépticas, o colo foi identificado pelo toque bidigital e o pólo cefálico elevado levemente para permitir a introdução da sonda. A mão utilizada para o toque bidigital serviu como guia para a introdução do cateter na cavidade uterina sob visão ultra-sonográfica (Aloka SSD-500 Micrus, Tóquio, Japão) até que a ponta do cateter estivesse posicionada próximo do pescoço fetal. A infusão inicial foi contínua e a velocidade de infusão para o primeiro frasco foi de 20 a $30 \mathrm{ml}$ por minuto, sendo reduzida para $3 \mathrm{ml}$ por minuto para os frascos seguintes. Toques vaginais foram realizados para elevar a apresentação fetal e permitir a saída do líquido. A infusão foi interrompida quando era percebida uma diluição do mecônio. A indicação de cesárea foi baseada em critérios obstétricos. O recém-nascido foi examinado pelo pediatra logo após o nascimento para identificar a presença de mecônio abaixo das cordas vocais. Os fetos foram acompanhados até 24 horas após o nascimento para identificação de sinais de disfunção respiratória, de acordo com os critérios pediátricos.

O tipo de parto realizado e a presença de eventuais complicações relacionadas com a amnioinfusão foram avaliados.

A privacidade e a autonomia das pacientes foi garantida de acordo com as questões éticas envolvidas.

\section{Resultados}

A idade das pacientes variou de 17 a 35 anos com média de idade de 27,35. A idade gestacional variou de 37 a 41 semanas com uma média de 39 semanas. O peso fetal variou de $2.965 \mathrm{~g}$ a $4.100 \mathrm{~g}$, tendo como média $3.489,9 \mathrm{~g}$ (Tabela 1 ). Uma média de $2.125 \mathrm{ml}$ de soro fisiológico foi infundida.

Tabela 1 - Caracterização do grupo de pacientes submetidas a amnioinfusão.

\begin{tabular}{lc}
\hline Fatores & Média \pm DP \\
\hline Idade (anos) & $27,35 \pm 1,05$ \\
Idade gestacional (semanas) & $39 \pm 0,27$ \\
Peso fetal (gramas) & $3.489,9 \pm 78,01$ \\
Volume de soro fisiológico infundido (ml) & $2.125 \pm 253$
\end{tabular}

Três pacientes demonstraram um aumento da intensidade da contratilidade uterina sem que houvesse complicações.

A incidência de parto cesáreo foi de $3 / 20$ (15\%). As indicações foram: um caso por desproporção céfalo-pélvica $(5 \%)$ e dois por sofrimento fetal agudo (10\%).

Nenhum feto apresentou mecônio abaixo das cordas vocais ou disfunção respiratória até a alta hospitalar. Não foram observadas complicações relacionadas com a utilização da técnica da amnioinfusão durante ou após o parto.

\section{Discussão}

A experiência internacional acumulada com o uso da amnioinfusão associada com a falta de relatos na literatura nacional sobre essa técnica motivaram a realização do presente estudo clínico.

O uso dessa técnica na literatura já está bem estabelecido ${ }^{2,3,4,8}$ e demonstrou ser importante para o manejo obstétrico ${ }^{9}$, apesar de que a sua utilidade não tenha sido confirmada em um estudo retrospectivo com mais de 900 pacientes de um 
hospital terciário ${ }^{10}$. Macri et $a .^{8}$, num estudo prospectivo randomizado com 170 pacientes em trabalho de parto e com mecônio espesso no líquido amniótico, verificaram que os neonatos que tiveram amnioinfusão apresentavam menos mecônio abaixo das cordas vocais (4 de 85), quando comparados com o grupo que não recebeu a amnioinfusão (33 de 85), apresentando um risco relativo de 0,12. Essa diferença também se verificou com respeito à presença da sindrome da aspiração do mecônio. Nenhum neonato apresentou a sindrome no grupo da amnioinfusão, ao passo que, no grupo sem a amnioinfusão, 5 de 85 a apresentaram (risco relativo de 0,09). Dye et al. ${ }^{4}$ demonstraram resultados semelhantes numa meta-análise com um total de 507 pacientes. Os neonatos tratados com amnioinfusão apresentavam uma redução média de $87 \%$ na apresentação de mecônio abaixo das cordas vocais. Esses dados são semelhantes aos observados no presente estudo, em que nenhum neonato apresentou mecônio abaixo das cordas vocais.

De acordo com a literatura, deve-se utilizar um cateter especial para a monitorização da pressão intra-uterina. Pelo fato de ser dificil de se encontrar esse dispositivo no nosso meio, foi decidido, no princípio, utilizar a sonda de Foley. Todavia, essa sonda se mostrou muito flexível, o que tornava dificil a sua introdução na cavidade uterina. Por isso, a sonda nasogástrica foi utilizada por ser mais rígida. Esse método demonstrou ser de fácil execução.

Para infusão, o soro fisiológico não precisou ser aquecido, como já havia sido demonstrado por Nageotte et al. ${ }^{6}$.

A velocidade inicial da infusão do soro fisiológico, no presente estudo, foi de 20 a $30 \mathrm{ml} /$ $\mathrm{min}$, cerca de $1.200 \mathrm{ml}$ a $1800 \mathrm{ml}$ por hora. Esse volume é maior do que o descrito na literatura revisada. Nesses estudos, a velocidade da infusão inicial variou de $500 \mathrm{ml}$ a $1.500 \mathrm{ml}^{4,8,10}$. Apesar de não haver um consenso sobre o volume a ser infundido, é provável que o volume intra-uterino que permaneceu da infusão inicial seja semelhante ao descrito na literatura, uma vez que a elevação da apresentação permitiu a saída do líquido infundido.

Com respeito às complicações observadas, três pacientes demonstraram um aumento da intensidade da contratilidade uterina após a infusão do soro fisiológico, o que poderia estar relacionado com a distensão das fibras uterinas. Esse fato também já foi relatado na literatura ${ }^{11}$. Usta et al. ${ }^{10}$ relatam o caso de uma paciente que apresentou dor abdominal devido à polidrâmnio agudo. O caso foi solucionado com a elevação da apresentação fetal e com a drenagem de mais de
$2.000 \mathrm{ml}$ de líquido claro.

O soro fisiológico foi escolhido como meio para infusão, mas pode-se utilizar o Ringer lactato, uma vez que já foi relatado que não existe diferença no resultado sobre as condições fetais de acordo com o tipo de soro infundido ${ }^{12}$.

Por ser uma técnica ainda não-empregada amplamente em nosso meio, algumas considerações devem ser feitas. A elevação da apresentação fetal para eliminar uma quantidade de líquido tem por objetivo reduzir a pressão intraamniótica e permitir que o líquido com mecônio tenha maior diluição. O perigo de prolapso de cordão não pode ser afastado; esse fato, felizmente, não foi verificado no presente trabalho. De forma semelhante, Macri et al. ${ }^{8}$, na sua casuística com 85 pacientes, não observaram nenhuma complicação com esse procedimento.

A utilização desse método nessa pequena amostra demonstrou ser segura e eficaz. Entretanto, existem complicações descritas na literatura, que apesar de raras, devem ser mencionadas. Usta et al. ${ }^{10}$ verificaram uma maior incidência de endometrite nas pacientes com amnioinfusão, quando comparadas com o grupo que não recebeu a infusão ( 21 versus $13 \%, \mathrm{p}=$ $0,004)$. Monahan et $a{ }^{13}$, por outro lado, num estudo prospectivo randômico, verificaram que a amnioinfusão reduz os casos de endometrite em pacientes de risco. A deiscência de cicatriz uterina em pacientes com cesárea prévia ${ }^{1,10}$, o prolapso de cordão ${ }^{1}$ e a embolia pulmonar por líquido amniótico ${ }^{14}$ foram descritos em casos esporádicos. Esse último evento é um acontecimento raro, por isso, é necessária a realização de estudos multicêntricos para confirmar essa associação.

Portanto, em razão de seu baixo custo e relativa segurança, seria adequada a realização de estudos prospectivos, controlados e randômicos em outras instituições brasileiras. Esses estudos poderiam ser conduzidos com o intuito de verificar se os índices de cesárea, de infecções pós-parto ou da síndrome de aspiração de mecônio nos recémnascidos são significativamente alterados com o uso da técnica da amnioinfusão dentro do contexto brasileiro.

\section{SUMMARY}

Purpose: to report the personal experience with the use of the amnioinfusion technique in patients in labor presenting meconial amniotic fluid, and the incidence of complications, the meconium aspiration syndrome and of cesarean sections. Method: twenty patients at term and in labor with meconial 
amniotic fluid were evaluated retrospectively, at the delivery ward at two public institutions of Rio Grande do Sul. An initial infusion of $1.000 \mathrm{ml}$ of normal saline solution at room temperature, at an infusion rate of 20-30 ml per minute was initiated and then reduced to $3 \mathrm{ml}$ per minute. The liquid was drained by elevating the cephalic pole.

Results: the procedure was feasible when a nasogastric catheter was used. None of the patients presented major complications related to the procedure. None of the neonates presented meconium below their vocal cords. The cesarean section rate was $3 / 20$ (15\%)

Conclusion: the amnioinfusion is a low-cost and feasible technique that did not show any complication in this study.

KEY WORDS: Amnioinfusion. Fetal distress. Labor.

\section{Referências}

1. Miyazaki FS, Taylor NA. Saline amnioinfusion for relief of variable or prolonged decelerations. A preliminary report. Am J Obstet Gynecol 1983; 146: $670-8$.

2. Schrimmer DB, Macri CJ, Paul RH. Prophylactic amnioinfusion as a treatment for oligohydramnios in laboring patients: a prospective randomized trial. Am J Obstet Gynecol 1991; 165: 972-5.

3. Miyazaki FS, Nevarez F. Saline amnioinfusion for relief of repetitive variable decelerations: a prospective randomized study. Am J Obstet Gynecol 1985; 153: 301-6.

4. Dye T, Aubry R, Gross S, Artal R. Amnioinfusion and the intrauterine prevention of meconium aspiration. Am J Obstet Gynecol 1994; 171: 1601-5.
5. Sokol RJ, Jones TB, Pernoll ML. Methods of Pregnancy Assessment for Pregnancy at Risk. In: DeCherney $\mathrm{AH}$, Pernoll ML, eds. Current Obstetric \& Gynecologic Diagnosis \& Treatment, 8th ed. New Jersey: Applenton \& Lange, 1994: p. 275-305.

6. Nageotte MP, Bertucci L, Towers CV. Prophylactic amnioinfusion in pregnancies complicated by oligohydramnios: a prospective study. Obstet Gynecol 1991; 77: 677-80.

7. Cecatti JG, Pires HMB, Aquino MMA. Amnioinfusão na prática obstétrica. Femina 1999; 27: 19-24.

8. Macri CJ, Schrimmer DB, Leung A. Prophylatic amnioinfusion improves outcome of pregnancy complicated by thick meconium and oligohydramnios. Am J Obstet Gynecol 1992; 167: $117-21$

9. Uhing MR, Bhat R, Philobos M, Raju TN. Value of amnioinfusion in reducing meconium aspiration syndrome. Am J Perinatol 1993; 10: 43-5.

10. Usta IM, Mercer BM, Aswad NK, Sibai BM. The impact of a policy of amnioinfusion for meconiumstained amniotic fluid. Obstet Gynecol 1995; 85: 237-41.

11. Posner MD, Ballagh SA, Paul RH. The effect of amnioinfusion on uterine pressure and activity: a preliminary report. Am J Obstet Gynecol 1990; 163: $813-8$

12. Puder KS, Sorokin Y, Bottoms SF. Amnioinfusion: does the choice of solution adversely affect neonatal electrolyte balance? Obstet Gynecol 1994; 84: 956-9.

13. Monahan E, Katz VL, Cox RL. Amnioinfusion for preventing puerperal infection. A prospective study. J Reprod Med 1995; 40 : 721-3.

14. Maher JE, Wenstrom KD, Hauth JC, Meis PJ. Amniotic fluid embolism after saline amnioinfusion: two cases and review of the literature. Obstet Gynecol 1994; 83: 851-4. 